\title{
Familiarity, relative distinctiveness, and the generation effect
}

\author{
ZEHRA F. PEYNIRCIOĞLU and ESRA MUNGAN \\ The American University, Washington, D.C.
}

\begin{abstract}
In Experiment 1, psychology experts and novices showed generation effects with both psychology. related and other words. In Experiment 2, music experts who were sports novices and sports experts who were music novices showed a generation effect in a recognition test for all words regardless of domain (music or sports). Moreover, the effect was greater for words from the subjects' "nonexpertise" area. In Experiments 3A and 3B, music experts showed a greater generation effect for sports words than for music words in a free recall test but only when the sports and music words were studied together. These results are inconsistent with the semantic elaboration requirement for the generation effect that predicts less of an effect, if any, with less familiar materials. Rather, they provide evidence for the idea that the generation effect is influenced by relative distinctiveness of the to-be-remembered items.
\end{abstract}

In memory experiments, items that are generated by the subjects themselves are remembered better than those that are presented by the experimenter (e.g., Slamecka $\&$ Graf, 1978). Many of the more popular explanations of this generation effect rely on associations between items and their representations in semantic memory. Recently, the idea of familiarity has emerged as a relevant dimension in the exploration of the boundary conditions of the generation effect. In particular, demonstrations of the effect with words but not with nonwords (e.g., McElroy \& Slamecka, 1982), with meaningful but not with meaningless letter bigrams, and with familiar but not with unfamiliar noun compounds (Gardiner \& Hampton, 1985), and with high-frequency words but not with low-frequency words-at least unfamiliar low-frequency words-(e.g., Nairne \& Widner, 1988) favor the conclusion that familiarity is a crucial factor that influences the generation effect.

A straightforward prediction from this view is that expertise or familiarity with a certain domain should affect the magnitude of the generation effect. Certainly, to the extent that memory performance benefits from organization, the existence of relevant schemata, meaningfulness, and knowledge-based strategies such as chunking, experts have a considerable advantage over novices in remembering items that are in their domain of expertise (e.g., Bartlett, 1932; Chase \& Simon, 1973; de Groot, 1966; Noble, 1963). Thus, experts should also show a greater generation effect for items in their domain of expertise, since they are said to have more "associative links" between these items or more "integrated conceptual representations" for these items (e.g., Gardiner, Gregg, \&

\footnotetext{
We thank D. Burns, J. Gardiner, J. Hampton, J. Nairne, and D. Payne for helpful comments on previous versions of this manuscript. Correspondence concerning this article should be sent to Zehra Peynircioglu, Department of Psychology, The American University, Washington, DC 20016.
}

Hampton, 1988; Nairne, Pusen, \& Widner, 1985). Indeed, Reardon, Durso, Foley, and McGahan (1987) have investigated whether experts, because they have a richer knowledge base, are more likely to engage in more effective conceptual processing of items in their fields of expertise and thus show a greater generation effect.

In Reardon et al.'s (1987) study, the experts were psychology faculty and graduate students, the novices were undergraduate students taking psychology courses, the critical area was psychology, and the control area comprised sports and leisure activities. Briefly, they found no generation effects in any of the conditions except with experts in the critical area. That is, there was no advantage of generating items over reading them for either group in the control area, and neither was there such an advantage for novices in the critical area. Thus, Reardon et al. concluded that the generation effect occurred only when the items were generated from a rich base of knowledge about such items.

Such a conclusion may seem attractive, because it is in line with the popular idea that semantic activation of conceptual representations and familiarity with the material are necessary for the generation effect to emerge. It may not be entirely correct, however, since most generation effect studies use words selected at random. In addition, there is evidence, albeit not yet overwhelming, that generation effects can arise, under some conditions, also with nonwords (Johns \& Swanson, 1988) and nonsense figures (Peynircioğlu, 1989). Thus, in Reardon et al.'s (1987) study, the lack of an effect in all conditions but the one involving the experts in their expertise area is somewhat surprising.

In the present study, we explore the effects of familiarity with the to-be-remembered material on the generation effect. First, we report an experiment similar to that of Reardon et al. (1987). Then, we will report an experiment in which instead of there being one critical area for 
which experts and novices existed and one control area that was assumed to be equally familiar to both groups (as in Experiment 1), there were two critical areas, and the experts in one acted as the novices in the other. We had music experts who were novices in sports and sports experts who were novices in music; and the study list comprised to-be-generated and to-be-copied words from both domains. Finally, we report two experiments in which free recall instead of recognition gauged memory performance, generation difficulty was manipulated during study, and the expertise (music) and novel (sports) area words were presented to musicians either in the same list or in separate lists.

\section{EXPERIMENT 1}

This experiment was similar to Reardon et al.'s (1987) study in that psychology experts and novices were presented with words of which some pertained to psychology and some to leisure activities. Of interest was whether psychology experts would show a greater generation effect with psychology words and whether the novices would show any effect with such words.

\section{Method}

Subjects. A total of 32 subjects at The American University participated in the experiment. Of these, 16 were psychology faculty and advanced (third year or above) graduate students and 16 were undergraduates who had taken two or more psychology courses. The undergraduates received credit in their psychology courses and faculty and graduate students were thanked for their efforts.

Materials, Design, and Procedure. The study list comprised 48 critical words and 12 buffer words (6 at each end, to attenuate primacy and recency effects). Of the critical words, 24 pertained to psychology and 24 to leisure activities. Half the words in each category were written at the end of descriptive sentences and were to be simply copied down (the read condition). The other half of the words were to be generated and written down with the help of the descriptive sentences and the first one to three of their letters (the generate condition). For example, the word reinforcement in the psychology category would be presented as "According to Skinner, a reward is a positive REINFORCEMENT' in the read condition and as "According to Skinner, a reward is a positive RE _ _ _ _ _ _ _ _ " in the generate condition; and the word neurons as "Cells of the nervous system are called NEURONS" in the read condition and as "Cells of the nervous system are called NE _ _ _ _ " in the generate condition. Similarly, the words camping and final in the "leisure" category would be presented as "If you don't like hotels, why not go CAMPING" and "The last match in most sports is called a FINAL" in the read conditions and as "If you don't like hotels, why not go CA _ _ _.." and "The last match in most sports is called a FI___." in the generate conditions. The words in the study list were presented in a mixed fashion with respect to both category (psychology or leisure) and generate-read condition. The presentation order of the study words was the same for all subjects, but across two subgroups of subjects, each word was presented in the generate and read conditions equally often. A free-choice recognition test followed the study list presentation and comprised 72 words, all of the critical 24 psychology and 24 leisure words that were studied (targets) plus 24 new words (lures), 12 of which were psychology words and 12 of which were leisure words. The testing order was the same for all subjects but it was different from the presentation order and mixed with respect to generate-read condition, type of category, and targetlure status.

The subjects were tested individually. They were first presented with the study words and their respective sentences on three sheets of paper. The sheets were initially covered, and the subjects uncovered each sentence and word at a rate of one item every $10 \mathrm{sec}$, paced by the experimenter. In keeping with Reardon et al.'s (1987) instructions, the subjects were told to rate each word after they had either generated or copied it in terms of how well-known the word would be for their peer group (on a 7-point scale, where 1 corresponded to very likely and 7 to very unlikely). They were also told about a memory test that would follow the presentation phase. Reardon et al. had not told their subjects about the memory test. Our purpose in changing the task to an intentional memory task was to impose even more stringent criteria for the occurrence of the generation effect, since expected memory tests often give rise to substantially smaller generation effects (Watkins \& Sechler, 1988). Another difference from Reardon et al.'s study was that since the subjects had to write down the generated words, we could see when they were not successful, and in such instances the target word was given to them in the same format as it was in the read condition. In the free-choice recognition test, each of the 72 words was printed on a $3 \times 5$ in. index card and presented in the same order to all the subjects. The subjects were given an otherwise blank sheet that contained the numbers 1-72; their task was to decide whether the word on each card had been studied or not by writing a " $Y$ " (for studied) or an " $\mathrm{N}$ " (for not studied) beside the corresponding number on the answer sheet.

\section{Results and Discussion}

The results are summarized in Table 1. Before turning to the results of main interest, however, we should mention that the false recognition rates did not differ for the two groups of subjects (overall, $3.7 \%$ for both groupsfor psychology experts, $5.2 \%$ for psychology words and $2.1 \%$ for leisure words, and for psychology novices, $4.2 \%$ for psychology words and $3.1 \%$ for leisure words) or for the two categories of words $(4.7 \%$ and $2.6 \%$ for psychology and leisure words, respectively) (all $p s>.05$ ). We should also mention that, consistent with previous literature on the role of expertise on memory performance (e.g., Chase \& Simon, 1973), overall, experts recognized more words from their area of expertise than from the other area (only 3 subjects recognized more leisure words); the undergraduates, on the other hand, did not systematically recognize any more words from a given area (6 recognized more leisure words, 5 recognized more psychology words, and 5 recognized equal numbers of leisure and psychology words).

As can be seen in Table 1, the generation effect emerged for both groups of subjects and both categories. In fact, both effects were statistically significant on a sign test. No expert (psychology faculty and advanced graduate students) and no nonexpert (undergraduates) recog-

Table 1

Mean Percentage of Correct Responses in Experiment 1

\begin{tabular}{lccccc}
\hline & \multicolumn{2}{c}{ Psychology Words } & & \multicolumn{2}{c}{ Leisure Words } \\
\cline { 2 - 3 } \multicolumn{1}{c}{ Subjects } & Generate & Read & & Generate & Read \\
\hline Psychology experts & 98.4 & 87.0 & & 94.3 & 81.8 \\
Psychology novices & 92.2 & 76.6 & & 91.7 & 72.4 \\
\hline
\end{tabular}


nized more of the read psychology words than generated psychology words, and only 2 experts and 1 nonexpert recognized more of the read leisure words than generated leisure words. More importantly, there was no indication that the generation effect was any greater for psychology words than for leisure words for either the psychology experts or the undergraduates $\left[F_{\mathrm{s}}(1,15)=.04\right.$ and .55 , $p \mathrm{~s}>.05$, and $M S_{\mathrm{e}} \mathrm{s}=99.24$ and 97.15 , respectively].

Thus, we found generation effects for leisure words for both groups of subjects. In addition, nonexperts showed a generation effect for psychology words, too. There may well have been ceiling effects at play; such effects, however, would have no bearing on the fact that generation effects did emerge for both types of words and for both groups. These results were inconsistent with those reported by Reardon et al. (1987). Since there were a number of differences between the two studies, there could be a few reasons why our results were also different. We think that perhaps the most likely reason is that although Reardon et al. checked for sentence completion performance at the end of the session (after the recognition test), since the generation efforts were initially covert, more novices may simply have failed to generate more of the psychology words during study. The initial generation failure rates in our study $(6.7 \%$ and $7.3 \%$ for leisure words and $3.6 \%$ and $15.1 \%$ for psychology words by psychology experts and novices, respectively) suggest such a possibility. At any rate, our results are inconsistent with the prediction that more familiar materials should lead to a greater generation effect.

\section{EXPERIMENT 2}

In the following experiment, we used a different design to address the role of familiarity in the generation effect. There were two groups of subjects; each group was an expert in one domain but unfamiliar with the expertise domain of the other group. Thus, one group comprised subjects who were experts in sports and novices in music, and the other group comprised subjects who were experts in music and novices in sports.

\section{Method}

Subjects. A total of 48 students at Bogaziçi University in Istanbul, Turkey, participated in the experiment for extra credit in psychology courses. The recruitment sign-up sheets asked specifically for experts in either classical music or sports (the subjects that were experts in both were not allowed to participate). A questionnaire about their expertise was given before the experiment. Each of the 24 subjects who were experts in music answered "yes" to questions such as "do you play an instrument?" and "can you read music and have you received any formal musical training?" Similarly, each of the 24 subjects who were experts in sports answered "yes" to questions such as "do you play or have you played any varsity or club team sports?" and "do you feel confident enough about your knowledge of soccer, basketball, and volleyball to be able to act as a TV commentator?"

Materials, Design, and Procedure. The method was similar to that of Experiment 1 . The words were 36 classical music and 36 sports terms (from soccer, basketball, and volleyball); 24 words from each category were randomly selected as the target words and assembled into a study booklet in an alternating fashion with respect to category. The remaining 24 words, 12 in each category, acted as lures in the subsequent recognition test. Half of the target items in each category were randomly assigned to the generate condition, and the other half to the read condition. As in the previous experiment, the study booklets contained one word (in the read condition) or word fragment whose first two letters were in place (in the generate condition) along with its descriptive sentence on each page. Some examples in the generate condition (with their descriptive sentences translated) are: (1) In soccer, when the goalie saves a shot by hitting it over the goal post, the other team earns a KO____ (corner kick); (2) Chopin's Etude Op. 10 No. 12 is written in F MI _ _ (minor); (3) If you hit a ball before it touches the ground, it is called a VO_- (volley); (4) Half a note above $\mathrm{C}$ is $\mathrm{C} \mathrm{DI}_{-}$- $_{-}$(sharp). In the read conditions, the descriptive sentences were the same but the words were already written in (e.g., KORNER, MINÖR, VOLE, and DIYEZ), and there were blanks immediately below each one (e.g.. _ $\ldots-\ldots-$ below KORNER) for subjects to copy it down. The words were presented in the same order to all subjects; across two groups of subjects for both kinds of experts, however, the generate and read conditions were interchanged, so that each word served in each condition equally often. The free-choice recognition test that followed included all 72 words typed on a single sheet; the presentation order was mixed with respect to study order, type of category, generate-read condition and target-lure status.

The subjects were tested individually or in small groups. As in Experiment 1, they were given a booklet with some sentences that were followed by either a word or a fragment of a word and asked to copy down the word or complete the fragment with the appropriate word. The purpose, they were told, was to gather some pilot data on the ease with which they could write words in their area of expertise as well as in the other area for a future experiment that would be used to test "general cultural knowledge" in a more sensitive way. Thus, unlike in the previous experiment, the memorial nature of the task was disguised.

During study, $10 \mathrm{sec}$ were allowed for reading each sentence and copying down the word or completing the word fragment. After all the words had been presented, a 15 -sec distractor task of counting backwards by threes was given to divert the subjects' attention from the last few words. Then the subjects were given a recognition test. They were told at that point that we were also interested in seeing how well they could remember the words they just saw. The recognition sheet contained all 72 words, and subjects indicated for each word whether it had been studied or not; this time, in addition to writing " $Y$ " or " $N$ " beside each word, they were also asked to give a confidence rating of 1 (guess), 2 (think so), or 3 (sure) for each decision. They were allowed 5 sec to work on each word, paced by the experimenter.

\section{Results and Discussion}

Although we used two measures to gauge memory performance (percentage of "yes" responses and recognition ratings that took into consideration the confidence ratings), we will report only the percentage of "yes" responses, since the patterns of results with the two measures were virtually identical. We would like to mention that when subjects failed to generate a word during study, they were not given the word as in Experiment 1, and the recognition test analyses we report were conditionalized on successful generation (generation failure rates for musicians and sports buffs were $3.5 \%$ and $5.6 \%$ for music words and $5.7 \%$ and $1.4 \%$ for sports words, respectively). We 
Table 2

Mean Percentage of Correct Responses in Experiment 2

\begin{tabular}{cccccc}
\hline & \multicolumn{2}{c}{ Music Words } & & \multicolumn{2}{c}{ Sports Words } \\
\cline { 2 - 3 } \cline { 5 - 6 } Subjects & Generate & Read & & Generate & Read \\
\hline Music experts & 96.5 & 80.2 & & 95.8 & 69.1 \\
Sports experts & 95.1 & 66.0 & & 94.8 & 74.0 \\
\hline
\end{tabular}

Table 3

Mean Percentage of Correct Responses in Experiment 2

\begin{tabular}{ccc}
\hline Area of Expertise & Generate & Read \\
\hline Own & 95.2 & 77.1 \\
Other & 95.5 & 67.5 \\
\hline
\end{tabular}

should note, however, that unconditionalized analyses, which were based on all test responses regardless of any generation failures during study, mirrored the results we report in every case. We would also like to mention that, overall, subjects falsely recognized $5.0 \%$ of the lure words although there were no reliable differences between those from the area of expertise and nonexpertise $[6.1 \%$ and $4.0 \%$, respectively, $t(47)=1.47, p>.10$ ].

The results are summarized in Tables 2 and 3 . Table 2 shows the responses of musicians and sports buffs separately, and Table 3 shows the responses of the two groups combined. As can be seen, overall, experts recognized more words from their expertise area than from the other area $\left[F(1,46)=9.77, M S_{\mathrm{e}}=30.09, p<.01 ; t(23)=\right.$ 2.37 for musicians and 1.94 for sports buffs, $p \mathrm{~s}<.05$, one-tailed]. Thus, as in Experiment 1, since "expertise" is a difficult concept to operationalize, it was helpful to observe that these results corroborated the self-reports of our subjects. It is also worth noting that this difference in "expertise" emerged because of the difference in performance for the words in the read conditions. In addition, since we were interested in the role of expertise or familiarity with the words rather than their general frequency of usage, we would like to note that the frequencies of our words varied over a great range. According to Thorndike and Lorge's (1944) norms, 6 of our sports words and 4 of our music words had frequencies of $A$ or AA (very common) and 5 of each category of words had frequencies of 17 per 4 million or lower (rather uncommon); the words with frequencies between these two endpoints also did not systematically differ in number in favor of either the music or the sports category. Thus, it appeared that the general frequencies of these words were roughly comparable, and the only difference was whether the words of one group were more or less familiar to the two groups of subjects.

Turning to the results of main interest, strong generation effects emerged in all conditions. Overall, in the subjects' expertise area, generated words were recognized better than read words; no subject recognized more read words than generated words. Likewise, in the subjects' nonexpertise area, only 2 out of the 48 subjects recognized more read than generated words. Also, not only did the generation effect emerge for words in the subjects' nonexpertise area, as well, but it was no less in magnitude, either. In fact, it was considerably enhanced in the nonexpertise area than in the expertise area $[F(1,46)=$ $10.76, M S_{\mathrm{e}}=101.67, p<.01 ; F(1,23)=6.23, M S_{\mathrm{e}}=$ 104.51 for musicians, and $F(1,23)=4.57, M S_{\mathrm{e}}=98.82$ for sports buffs, both $p s<.05$ ]

It may be argued that ceiling effects were again playing a role and that the overall rather high levels of recognition in the generate condition prevented the experts from showing more of an advantage in their domains of expertise than in the other domain. Since the response scale was, in effect, a continuous one from "No 3" to "Yes 3," one can use a stringent criterion and consider only the "Yes 3" responses as "true recognition" (i.e., when subjects said that the item was studied and they were sure). When these responses are considered, a similar pattern of results emerges. The mean percentages of correct responses in the subjects' domain of expertise were 90.6 for generated items and 68.4 for read items (91.3 to 67.0 for sports buffs and 89.9 to 69.8 for music buffs) and those in the other domain were 87.7 for generated items and 58.4 for read items (86.8 to 61.5 for sports buffs and 88.5 to 55.2 for music buffs). And the statistical tests mirror the results reported with mean percentage of correct responses. Thus, the greater generation effect obtained in the nonexpertise domain does not appear to be an artifact of ceiling effects. The mere emergence of the effect with nonexpertise domain items is again inconsistent with Reardon et al.'s (1987) findings, and the greater generation effect observed for words in the less familiar domain is inconsistent with the prediction that more familiar items should lead to a greater generation effect.

\section{EXPERIMENTS 3A AND 3B}

Although analyses with "Yes 3" responses somewhat alleviated the probability of ceiling effects' contaminating the comparisons in Experiment 2, we decided to address the problem more directly. Thus, instead of a recognition test, a free-recall test was used and the list was lengthened by the inclusion of many filler words at the end. Moreover, in the following two experiments, we looked at whether the magnitude of the generation effect depended on the difficulty of the generation process. One explanation of a greater generation effect with less familiar materials obtained in Experiment 2 might be that although subjects were given the same amount of time to study each word, they might have used up more of that interval to generate the less familiar words, thus creating, in effect, unequal study times.

\section{Method}

In Experiment 3A, the study list comprised 84 words. Half of these were related to classical music, and the other half to football, baseball, basketball, and tennis. The first 64 were critical words, and the last 20 were filler words to lengthen the list. Of the 32 critical words in each category (music or sports), 16 were in the generate condition and 16 in the read condition. Furthermore, in each cate- 
gory, 8 of the words in the generate condition were in the difficultgenerate condition and 8 in the easy-generate condition. The difficulty was defined in terms of fragment size. The easy fragments included more letters in addition to all of the letters included in their difficult counterparts. For instance, the word allegro was given as "it means play it fast: $A L_{-}$GRO" (the easy-generate condition) or "AL_ _ _ O" (the difficult-generate condition); and the word fumble was given as "in football, when one drops the ball, it is a FU_BLE or $\mathbf{F}_{-}{ }_{-} \mathbf{L}_{-}$." 'Across four groups of subjects, each word appeared in the read and generate conditions as well as in the easy- and difficult-generate conditions equally often.

The descriptive sentences and the to-be-read words or to-becompleted fragments were typed on sheets of paper. Each word or fragment had a box with two slots next to it. The words were presented at a 6-sec rate. The read words were copied in the first slot of the box. The generated words were written in the first slot of the box if they were generated in the first $3 \mathrm{sec}$ of the interval and in the second slot if they were generated in the second $3 \mathrm{sec}$ The instructions were illustrated by a few examples of each kind of word. The presentation of the words was paced by the experimenter who also indicated the 3-sec slices when relevant. After all 64 words were studied in this way, the subjects went through the filler words typed with no descriptive sentences at the end of the study list and simply copied them down. They knew they would not have to recall these later on. The presentation phase was directly followed by a 10-min free-recall test, and the subjects were asked to write down any word they could remember in any order they wished.

Experiment 3B was identical to Experiment 3A in every respect, except that the music words and the sports words were presented in two separate lists of 32 words rather than one mixed list of 64 words. Also, since the lists were shorter, the filler words at the end of each list were increased to 30 words of the same category. With eight groups of subjects, the order of list presentation, the generate-read conditions, and the easy- and difficult-generate conditions were counterbalanced.

The subjects were 20 and 32 music experts in Experiments $3 \mathrm{~A}$ and $3 B$, respectively, who had very little knowledge of sports and who were recruited primarily from the music department at The American University. The minimum criterion for expertise was 3 years of theoretical or instrumental instruction. They were tested individually or in small groups.

\section{Results and Discussion}

The results are summarized in Table 4 . Before turning to the results of main interest, we would like to note that overall generation failure rates were $0.8 \%$ and $3.5 \%$ for music and sports words, respectively, in Experiment 3A, and $1.4 \%$ and $5.1 \%$ in Experiment $3 B$. Similarly, consistent with their claims of expertise and the results of Experiment 2, the subjects recalled more music words than sports words in the read conditions.

In both experiments, strong generation effects emerged for both music (expertise area) and sports (nonexpertise area) words (all $p \mathrm{~s}<.01$ ). Moreover, in Experiment 3A,

Table 4

Mean Percentage of Correct Responses in Experiments 3A and 3B

\begin{tabular}{lccccc} 
& \multicolumn{2}{c}{ Music Words } & & \multicolumn{2}{c}{ Sports Words } \\
\cline { 2 - 3 } \cline { 5 - 6 } Experiment & Generate & Read & & Generate & Read \\
\hline 3A (mixed list) & 38.1 & 25.9 & & 38.1 & 15.9 \\
3B (pure list) & 53.7 & 29.3 & & 54.3 & 26.0 \\
\hline
\end{tabular}

when all the words were presented in a mixed list, the generation effect tended to be stronger for sports words $\left[F(1,18)=3.23, M S_{\mathrm{e}}=154.61, p<.09\right]$. There was no hint of such an interaction in Experiment 3B $[F(1,30)$ $\left.=.99, M S_{\mathrm{e}}=122.23, p>.10\right]$.

Also, in neither experiment did the difficulty of the cues appear to affect the magnitude of the generation effect ( $p$ s $>.10$ in both experiments), although subjects did, on the average, take a longer time with the words in the difficult-generate condition during the study phase. Only $5.4 \%$ of the words in the easy-generate condition were discovered in the second slot $(1.6 \%$ for music words and $9.1 \%$ for sports words), whereas this percentage was $27.3 \%$ in the difficult-generate condition $(18.0 \%$ for music words and $36.6 \%$ for sports words). Thus, consistent with findings showing the magnitude of the generation effect to be relatively independent of the generation rule (e.g., Jacoby, 1978; Slamecka \& Fevreiski, 1983; Slamecka \& Graf, 1978; but see Gardiner, Smith, Richardson, Burrows, \& Williams, 1985, for an exception), it seems that greater effort or elaboration is not a likely explanation for the trend for a greater nonexpertise area generation effect observed in the mixed-list experiment.

Generation effects emerged in all cases, regardless of area of expertise. In fact, consistent with findings from Experiment 2, the generation effect tended to be greater for nonexpertise area words when the expertise and nonexpertise area words were presented in a mixed fashion. The lack of a greater effect with nonexpertise area words when presented separately from the expertise area words could reflect the lack of competition between the words from the two areas and thus the dissipation of the distinctiveness the nonexpertise area words enjoyed in the mixed list. Such a finding is consistent with a relative distinctiveness idea.

\section{GENERAL DISCUSSION}

A popular construct in explaining the generation effect is a greater elaboration of the conceptual representations of self-generated items. From this construct, along with the assumption that a richer knowledge base would facilitate elaboration (cf. explanations of the effect of expertise on memory), one prediction is that when the conceptual representations of the target items are indeed from a richer knowledge base, the generation effect should be enhanced. Such a prediction is also consistent with the idea that familiarity plays a crucial role in the emergence of the generation effect, regardless of the particular explanation used (cf. Gardiner et al., 1988).

In the present study, we used a procedure similar to that used in Reardon et al.'s (1987) study and looked at the role of "expertise" or familiarity in the generation effect. They found a generation effect only when experts were presented with items from their expertise area; they found no such effect when novices were presented with the same items or when the subjects were presented with 
items from an area no one in the experiment was an expert in. With a similar design in Experiment 1, we found healthy generation effects for both experts and novices, regardless of type of word. Moreover, the magnitude of the effect was no greater in any particular condition than in any other.

In Experiments 2 and $3 \mathrm{~A}$, too, we found strong generation effects in both the area of expertise and the area of nonexpertise. In fact, the generation effect in the nonexpertise area was greater in these experiments. Music experts showed a greater effect with sports terms than with music terms, and sports experts showed a greater effect with music terms than with sports terms.

It appears that ease of semantic elaboration of the conceptual representations of items is not necessary for the generation effect. In fact, although music words were probably more familiar to musicians than were sports words and hence more readily integrated or associated with other words, there emerged a greater generation effect with the less familiar sports words in Experiments 2 and 3A. Similarly, in Experiment 2, sports buffs showed a greater generation effect with less familiar and thus presumably less integrable or associable music words than with more familiar sports words. Although lack of familiarity with certain words may have lessened the degree to which they could be elaborated on, it might also have given these words more of a chance to increase their distinctiveness through generation in comparison with similar read words during study. The performance with the words in the read conditions did appear to reflect the effect of familiarity; familiar read words were better remembered than less familiar read words. The words in the generate conditions, on the other hand, were equally likely to be remembered, regardless of familiarity. Thus, one might say that unfamiliar words benefit more from the act of generation than do familiar words, because the former are not very distinctive to begin with.

It is also interesting that when familiar and unfamiliar words are presented in separate lists, although generation effects again emerge for both, the effect with unfamiliar words, although slightly greater than that with familiar words, is not significantly so. Given that we assume memory performance on the read words to set the baseline for familiarity and general memorability, it appears that when they are presented alone, unfamiliar words do not benefit from the act of generation much more than do familiar words. We would like to note that, if pushed enough, there might have emerged a greater generation effect for unfamiliar words even in pure lists. But, at least, with the present results, it appears that the unfamiliar words need to be studied in the context of familiar words for their relative distinctiveness to be a significant factor. That is, when words are presented in a pure list, the act of generating a word increases its memorability compared to the act of simply reading it; but without direct competition between the two categories of words, this increase is roughly the same for both familiar and unfamiliar words, since it reflects the increase in distinctiveness rel- ative to the words in the same category. When words are presented in a mixed list, however, generating an unfamiliar word increases its distinctiveness in comparison with reading a similar word to a greater extent than does generating a familiar word.

Recently, Begg, Snider, Foley, and Goddard (1989) have proposed that the generated items are remembered better because they are made more distinctive in the list in comparison with the read words of the same sort. Such a proposal explains why generation effects are larger in mixed-list designs and why generating a word can hurt memory if the words are tested in a way that requires discriminations that rely on different types of processing than those at study. Begg et al. have also emphasized that the distinctiveness that the generated words enjoy depends on the context in which they are encountered; they have given the pretty example of a black cat being distinct against a snowdrift but not against a coal bin. Our results are consistent with such a proposal. When presented in the same list, generated expertise words gain a certain amount of distinctiveness in comparison with read expertise words and likewise with generated and read nonexpertise words. But the distinctiveness that the generated nonexpertise words gain appears to be greater than that for generated expertise words. Thus, in addition to the item-specific benefits enjoyed by the generated words, the categories of words, whether they are highly or not at all familiar, influence the magnitude of the gained distinctiveness. Since such an interaction occurs only when the expertise and nonexpertise words are presented together, one could perhaps push Begg et al. 's black cat example a little further and say that while a black cat against a snowdrift is more distinct than a black cat against a coal bin, this difference might be less striking than the difference between a green iguana (a less familiar beast) against a snowdrift than a green iguana against a grassy area when all are visible at the same time. Although we have not demonstrated relational benefits of generating in the absence of item-specific benefits, we have demonstrated that context-target relations (in this case influenced by familiarity) can be a factor beyond simply aiding in item-specific benefits in the generation effect.

Two other recent theories of the generation effect are Hirshman and Bjork's (1988) and McDaniel, Waddill, and Einstein's (1988) two- and three-factor theories, respectively. One of the factors in these theories is item-specific elaboration and hence pertains to the distinctiveness that Begg et al. (1989) were suggesting. The second factor common to both theories is cue-target relational processing. The relationship between the cue word and the generated response word is important, especially in structured lists such as ours (as the third factor-overall list structure and organization-in McDaniel et al.'s theory suggests). The implication is that the stronger the relationship, the more of a benefit this will be for future remembering as long as the type of test makes use of processes congruent with the relational processing engaged in during study. One assumption is that the more familiar the cue and tar- 
get words, the easier it should be to engage in such relational processing. This assumption is indeed in line with other explanations of the generation effect that rely on the idea of conceptual elaboration of already existing concepts (e.g., McElroy \& Slamecka, 1982).

Our findings also suggest that the cue-target relationship is an important factor but for a different reason. In the present case, this importance appears to be at least partially due to enhancing the relative distinctiveness of the words. In our experiments, the less familiar words, and thus presumably the pairs with the less strong relationships, showed greater generation effects. One could speculate that for the words that were highly familiar, the cue-target relationships were more commonly encountered ones, and thus, though strong, were not as salient an attribute as the cue-target relationships between words that were highly unfamiliar. And one could speculate that the unfamiliar and thus less known cue-target relationships served to enhance the distinctiveness of the target words. Thus, we agree with Begg et al.'s (1989) proposal that it is the distinctiveness gained by the act of generation that renders those words more memorable. We also agree with Hirshman and Bjork (1988) and McDaniel et al. (1988) that relational processing is important, too, but in some cases by virtue of adding to the distinctiveness gained by generating. In this sense, it is the relative distinctiveness that appears to combine the two factors.

In the same vein, we did not get a bigger effect with psychology words than with everyday leisure words for psychology novices in Experiment 1, even though both types of words were studied in the same list. We suspect that this was because the discrepancy in familiarity for the two types of words for our novices was not as great as that between familiarity for words in one's special area of expertise and those in an area one has no interest or knowledge in; thus, the additional distinctiveness provided by unfamiliarity of the words was not large enough. Again, the main idea is relative distinctiveness.

We would like to note that, as we have mentioned earlier, familiarity, though related to word frequency, is not synonymous with word frequency. For instance, the words pass, yard, or serve are probably more commonly encountered even by music experts than the words baritone, metronome, or virtuoso, although, in their contexts in the study list, the latter words are probably more familiar to musicians who do not know much about sports. Thus, perhaps a minimal degree of acquaintance with the items is necessary for distinctiveness to show its effect; nonwords and low-frequency words sometimes do not show the generation effect (e.g., McElroy \& Slamecka, 1982; Nairne et al., 1985; but see, e.g., Gardiner et al., 1988, for an exception). Once the items are "frequent enough," however, it appears to be their relative distinctiveness that influences their memorability and the generation effect. Gardiner and Hampton (1988) have eliminated the generation effect by introducing an orienting task that presumably reduced the relative distinctiveness that the generated items usually enjoy. In the absence of any other manipulation, items that are less familiar appear to benefit more from generation, thus creating a wider gap between these words and their read counterparts in terms of distinctiveness.

Our purpose was not to provide support for or against any particular theory of the generation effect. Specific mechanisms of each such theory notwithstanding, the idea of familiarity is assumed to play a role in most of these theories. In most cases, the assumption is roughly that familiarity influences the degree of semantic elaboration or relational processing that can be engaged in to promote the generation effect. Thus, the prediction is that greater generation effects will occur with more familiar materials. This was not the case in our study. Slightly elaborating on Begg et al.'s (1989) proposal, we believe that it was the greater relative distinctiveness of the less familiar words that gave rise to a greater generation effect with these words; the act of generation increased the distinctiveness of such words in comparison with their read counterparts to a greater extent than it did the distinctiveness of more familiar words.

\section{REFERENCES}

Bartlett, F. C. (1932). Remembering: A study in experimental and social psychology. Cambridge: Cambridge University Press

Begg, I., Snider, A., Foley, F., \& Goddard, R. (1989). The generation effect is no artifact: Generating makes words distinctive. Journal of Experimental Psychology: Learning, Memory. \& Cognition, 15, 977-989.

Chase, W. G., Simon, H. A. (1973). Perception in chess. Cognitive Psychology, 5, 55-81.

DE Groot, A. (1966). Perception and memory versus thought: Some old ideas and recent findings. In B. Kleinmuntz (Ed.), Problem solving: Research, method and theory (pp. 19-50). New York: Wiley.

Gardiner, J. M., Gregg, V. H., Hampton, J. A. (1988). Word frequency and generation effects. Journal of Experimental Psychology: Learning, Memory, \& Cognition, 14, 687-693

Gardiner, J. M., \& Hampton, J. A. (1985). Semantic memory and the generation effect: Some tests of the lexical activation hypothesis. Journal of Experimental Psychology: Learning, Memory, \& Cognition, 11, 732-741.

Gardiner, J. M., \& Hampton, J. A. (1988), Item-specific processing and the generation effect: Support for a distinctiveness account. American Journal of Psychology, 101, 495-504.

Gardiner, J. M., Smith, H. E. C., Richardson, C. J., Burrows, M. V., \& Williams, S. D. (1985). The generation effect: Continuity between generating and reading. American Joumal of Psychology, 98, 373-378.

Hirshman, E., \& Bjork, R. A. (1988). The generation effect: Support for a two-factor theory. Joumal of Experimental Psychology: Leaming. Memory, \& Cognition, 14, 484-494

JACOBY, L. L. (1978). On interpreting the effects of repetition: Solving a problem versus remembering a solution. Joumal of Verbal Learning \& Verbal Behavior, 17, 649-667.

Johns, E. E., \&WANSON, L. G. (1988). The generation effect with nonwords. Journal of Experimental Psychology: Leaming, Memory, \& Cognition, 14, 180-190.

McDaniel, M. A., Waddill, P. J., \& Einstein, G. O. (1988). A contextual account of the generation effect: A three-factor theory. Journal of Memory \& Language, 27, 521-536.

McElroy, L. A., \& Slamecka, N. J. (1982). Memorial consequences of generating nonwords: Implications for semantic-memory interpretations of the generation effect. Journal of Verbal Learning \& Verbal Behavior, 21, 249-259. 
Nairne, J. S., Pusen, C., \& Widner, R. L., JR. (1985). Representation in the mental lexicon: Implications for theories of the generation effect. Memory \& Cognition, 13, 183-191.

NAIRNe, J. S., \& WIDNER, R. L., JR. (1988). Familiarity and lexicality as determinants of the generation effect. Journal of Experimental Psychology: Learning, Memory, \& Cognition, 14, 694-699.

Noble, C. E. (1963). Meaningfulness and familiarity. In C. N. Cofer \& B. S. Musgrave (Eds.), Verbal behavior and learning (pp. 76-119). New York: McGraw-Hil].

Peynircioglu, Z. F. (1989). The generation effect with pictures and nonsense figures. Acta Psychologica, 70, 153-160.

Reardon, R., Durso, F., Foley, M. A., \& McGahan, J. R. (1987). Expertise and the generation effect. Social Cognition, 5, 336-348.

Slamecka, N. J., \& Fevreiski, J. (1983). The generation effect when generation fails. Journal of Verbal Learning \& Verbal Behavior, 22 , 153-163.

Slamecka, N. J., \& Graf, P. (1978). The generation effect: Delineation of a phenomenon. Journal of Experimental Psychology: Human Learning \& Memory, 4, 592-604.

Thorndike, E. L., \& LORGE, I. (1944). The teacher's word book of 30,000 words. New York: Teachers College Press, Columbia University.

Watkins, M. J., \& SeChler, E. S. (1988). Generation effect with an incidental memorization procedure. Journal of Memory \& Language, 27, 537-544.

(Manuscript received February 18, 1992;

revision accepted for publication September 25, 1992.) 